\title{
Registro colombiano de falla cardiaca (RECOLFACA): metodología y datos preliminares
}

\section{Colombian heart failure registry (RECOLFACA): methodology and preliminary data}

\author{
Juan E. Gómez-Mesa ${ }^{1 *}$, Clara I. Saldarriaga ${ }^{2}$, Luis E. Echeverría ${ }^{3}$, Paula Luna ${ }^{4}$ y \\ Grupo Investigador RECOLFACA
}

${ }^{1}$ Servicio de Cardiología, Fundación Valle del Lili, Cali; ${ }^{2}$ Servicio de Cardiología, Clínica Cardio VID, Medellín; ${ }^{3}$ Servicio de Cardiología, Fundación Cardiovascular de Colombia, Bucaramanga; ${ }^{4}$ Sociedad Colombiana de Cardiología, Bogotá. Colombia

\section{Resumen}

El registro RECOLFACA es la primera aproximación que se realiza en Colombia sobre el perfil clínico y demográfico de los pacientes con falla cardiaca, e incluye instituciones de gran parte del territorio nacional. Esta información es de gran relevancia ya que permitirá caracterizar la población y servir como base para diseñar políticas públicas que ofrezcan una mejor atención en salud a esta población. Este importante proyecto epidemiológico, llevado a cabo por el Capítulo de Falla Cardíaca, Trasplantes e Hipertensión Pulmonar de la Sociedad Colombiana de Cardiología y Cirugía Cardiovascular, es pionero en la evaluación de las enfermedades cardiovasculares en Colombia y, adicionalmente, servirá como modelo para la evaluación de otras enfermedades.

Palabras clave: Falla cardiaca. Hospitalización. Mortalidad. Tratamiento.

\begin{abstract}
The RECOLFACA registry is Colombia's first approach to describing the clinical and demographic profile of patients with heart failure, which includes institutions throughout much of the country. This information is of great importance as it will characterize the population and serve as the basis for designing public policies which will provide better health care to this population. This important epidemiological project, carried out by the Heart Failure, Transplants and Pulmonary Hypertension Chapter of the Sociedad Colombiana de Cardiología y Cirugía Cardiovascular [Colombian Society of Cardiology and Cardiovascular Surgery] is a pioneer in evaluating cardiovascular diseases in Colombia, and will also serve as a model for evaluating other diseases.
\end{abstract}

Key words: Heart failure. Hospitalization. Mortality. Treatment.

\section{Correspondencia:}

*Juan E. Gómez-Mesa

E-mail: juan.gomez.me@fvl.org.co
Disponible en internet: 29-07-2021

Rev Colomb Cardiol. 2021;28(3):217-230

www.rccardiologia.com 0120-5633 / @ 2020 Sociedad Colombiana de Cardiología y Cirugía Cardiovascular. Publicado por Permanyer. Este es un artículo open access bajo la licencia CC BY-NC-ND (http://creativecommons.org/licenses/by-nc-nd/4.0/). 


\section{Introducción}

La falla cardiaca es un problema de salud pública mundial que afecta a más de 23 millones de personas. Las cifras en los Estados Unidos de Norteamérica estiman 5.1 millones de afectados según el registro NHANES (National Health and Nutrition Examination Survey), y se espera que su prevalencia se incremente un 46\% del año 2012 al año 2030. En contraste con otras enfermedades cardiovasculares, la prevalencia, la incidencia y la mortalidad por falla cardiaca están en aumento ${ }^{1,2}$.

Después del diagnóstico clínico de falla cardiaca, los pacientes son hospitalizados en promedio 0.87 veces al año, es decir, casi una vez por año ${ }^{3}$. La morbilidad y la mortalidad generadas por la falla cardiaca tienen grandes repercusiones económicas en el mundo. En los Estados Unidos de Norteamérica, durante el año 2012, el costo total de la falla cardiaca se estimó en $\$ 30.7$ millones de dólares ${ }^{4}$. Después del egreso hospitalario por falla cardiaca agudamente descompensada, hasta un $60 \%$ de los pacientes deben ser rehospitalizados antes de 6 meses.

La mortalidad asociada a falla cardiaca es muy elevada, y es aún mayor en los pacientes de más de 65 años de edad. Se calcula una mortalidad a 8 años del $80 \%$ en los hombres y del $70 \%$ en las mujeres en este grupo $^{5-9}$. Sin embargo, si los pacientes requieren hospitalización por falla cardiaca agudamente descompensada la mortalidad es mucho mayor: el $3-10 \%$ de estos pacientes fallecen dentro del hospital, el 10-20\% fallecen en los primeros 30-60 días del egreso y el 50\% fallecen en los primeros 6 meses después del egreso $0^{5,6,9-12}$.

Los estudios acerca de la epidemiología en América Latina muestran que la prevalencia de la falla cardiaca está en aumento debido al incremento de los factores de riesgo cardiovasculares en la población general, la mayor tasa de sobrevida a los eventos isquémicos cardiovasculares y el incremento en la expectativa de vida $^{13}$. En Colombia, según datos del Ministerio de Protección Social, la principal causa de mortalidad es la enfermedad cardiovascular, la cual afecta principalmente a los grupos poblacionales mayores de 45 años. Entre las principales causas de esta elevada mortalidad están la enfermedad coronaria y la hipertensión arterial, que como hemos descrito anteriormente son los mayores condicionantes para desarrollar falla cardiaca. Según el reporte del Ministerio de Protección Social para el año 2011, la tasa de mortalidad por falla cardiaca era de 6.40 por 100.000 habitantes $^{14}$.
En Colombia se ha intentado describir las características clínicas y epidemiológicas de los pacientes con falla cardiaca. En 2011 se realizó un estudio que buscó establecer el perfil clínico de los pacientes con falla cardiaca agudamente descompensada que ingresan al servicio de urgencias $^{15}$, y en 2017 se describieron estos perfiles clínicos y hemodinámicos en pacientes con falla cardiaca agudamente descompensada ${ }^{16}$. Ningún registro previo incluyó cohortes de pacientes con falla cardiaca agudamente descompensada de instituciones de todo el territorio nacional y con seguimiento a largo plazo para poder conocer desenlaces clínicos, funcionales, mortalidad y rehospitalización. Por ello, el Registro Colombiano de Falla Cardíaca (RECOLFACA) pretende evaluar las características demográficas, clínicas y paraclínicas de los pacientes con falla cardiaca agudamente descompensada. Además, incluirá información sobre factores de riesgo, comorbilidad y condiciones desencadenantes relacionados con la falla cardiaca agudamente descompensada, al igual que de la evolución clínica de los pacientes hospitalizados y durante su manejo ambulatorio posterior, incluyendo información relacionada con el tratamiento, las complicaciones y la carga social de la falla cardiaca. Con estos datos sobre la realidad de la falla cardiaca en Colombia se busca generar políticas públicas para el diagnóstico y el tratamiento oportuno de esta enfermedad.

\section{Método}

Este estudio de cohorte prospectivo y multicéntrico fue diseñado por los miembros del Capítulo de Falla Cardíaca, Trasplante Cardíaco e Hipertensión Pulmonar de la Sociedad Colombiana de Cardiología y Cirugía Cardiovascular (SCC CCV). El comité coordinador del estudio estaba conformado por los doctores Juan Esteban Gómez, Clara Saldarriaga y Luis Echeverría. La coordinadora nacional fue la enfermera Paula Luna (SCC CCV). El comité administrativo estuvo integrado por Adriana Cruz y Diana Baquero (SCC CCV). La plataforma de recolección de datos y soporte electrónico estuvo a cargo de Jorge Bolaños (INFAMED).

El comité coordinador y la coordinadora nacional fueron los encargados de supervisar, monitorizar y coordinar el reclutamiento adecuado de pacientes, mientras que la coordinadora nacional fue la encargada de revisar la calidad de los datos ingresados y el correcto seguimiento de los pacientes reclutados. 


\section{Criterios de inclusión}

- Paciente mayor de 18 años.

- Antecedente de hospitalización por falla cardiaca en los últimos 12 meses.

- Paciente que asiste a consulta programada de seguimiento para falla cardiaca (supervisada y coordinada por médico internista, cardiólogo o especialista en falla cardiaca).

\section{Criterios de exclusión}

- Paciente con antecedente de trasplante cardiaco, en lista activa de trasplante cardiaco o en trámites administrativos para ingresar a lista activa de trasplante cardiaco.

- Paciente con implante previo de un dispositivo de asistencia ventricular o en proceso de implante de un dispositivo de asistencia ventricular.

- Incapacidad para cumplir los seguimientos programados (telefónicos o presenciales) por condiciones sociales, personales u otras, según considere el investigador principal.

- Limitación neurológica que afecte la adherencia y el seguimiento farmacológico, según considere el investigador principal.

- Limitación social que afecte la adherencia y el seguimiento farmacológico, según considere el investigador principal.

\section{Mediciones}

Los pacientes incluidos en el estudio debían completar una fase de reclutamiento y una fase de seguimiento a los 6 meses después del ingreso; esta visita de seguimiento podía ser presencial o telefónica.

- La primera fase del estudio se realizó en 2017 y 2018, y la segunda fase en 2018 y 2019. Cada una de estas fases incluía reclutamiento (ingreso en la base de datos) y seguimiento, el cual se realizaba 6 meses después del reclutamiento y podía hacerse de manera presencial, telefónica o mediante información obtenida en la historia clínica para ese periodo (6 meses). Las variables consideradas fueron: Variables sociodemográficas: edad, sexo, raza, estado civil, nacionalidad, municipio, departamento y área de residencia, escolaridad, régimen de seguridad social e institución médica donde el paciente recibe la atención.

- Variables clínicas: antecedentes patológicos, signos y síntomas detectados relacionados con la falla cardiaca (disnea paroxística, disnea de esfuerzo, doIor torácico, derrame pleural, taquicardia, edema de miembros inferiores, entre otros), hospitalizaciones por falla cardiaca y por otras causas, clase funcional de la New York Heart Association (NYHA), clasificación por estadios según el American College of Cardiology y la American Heart Association (ACC/AHA), fracción de eyección del ventrículo izquierdo (FEVI), etiología de la insuficiencia cardiaca, tratamiento farmacológico, presencia de dispositivos, presentación de Stevenson, factores de descompensación, presión arterial sistólica, frecuencia cardiaca, detalles de la radiografía de tórax, electrocardiograma y ecocardiograma, calidad de vida (EuroQol-5D), asistencia a un programa hospitalario de falla cardiaca y tipo de seguimiento recibido, y tratamiento farmacológico actual.

- Variables de laboratorio clínico: hemoglobina ( $\mathrm{g} / \mathrm{dl})$, creatinina $(\mathrm{mg} / \mathrm{dl})$, nitrógeno ureico en sangre $(\mathrm{mg} / \mathrm{dl})$, tasa de filtración glomerular $\left(\mathrm{ml} / \mathrm{min} / 1.73 \mathrm{~m}^{2}\right)$, electrolitos séricos (potasio y sodio, $\mathrm{mmol} / \mathrm{l}$ ), glucemia $(\mathrm{mg} / \mathrm{dl})$, péptido natriurético tipo $\mathrm{B}(\mathrm{pg} / \mathrm{ml})$ y propéptido natriurético tipo $\mathrm{B}$ fragmento $\mathrm{N}$ terminal $(\mathrm{pg} / \mathrm{ml})$.

\section{Registro de los datos}

Los centros médicos registraron sus datos de forma independiente en una plataforma de registro diseñada para dicha labor y mediante un formato de transferencia electrónica en la web. Posteriormente, la información fue exportada a una base de datos central, siguiendo el estricto programa de transferencia electrónica, para conformar así un registro común de acceso restringido en Excel. Previo al análisis, se realizó una depuración de la base de datos, y se verificaron y corrigieron los valores extremos y atípicos o sospechosos de errores contactando directamente con la institución que generó el registro.

\section{Análisis estadístico}

Se realizó un análisis netamente descriptivo de las características sociodemográficas y clínicas a línea de base de la cohorte. Las variables cualitativas se describen con valores absolutos y relativos (porcentajes). La normalidad de las variables cuantitativas fue explorada gráficamente por medio de histogramas y la prueba de normalidad de Shapiro Wilk; las variables con una distribución normal se presentan como media y desviación estándar, y de lo contrario se informan la mediana y los cuartiles primero y tercero (Q1-Q3). 
Todos los análisis se realizaron en el software estadístico Stata versión 14.0.

\section{Aspectos éticos}

Según la resolución 008430 de 1993 del Ministerio de Salud de Colombia, esta fue una investigación sin riesgo, dado que recolectó información de las historias clínicas y de las pruebas sistemáticas en esta población, sin generar procedimientos adicionales por fuera de la práctica diaria del manejo de los pacientes con falla cardiaca. Previo a su ejecución, el protocolo de investigación fue aprobado por el comité de ética de cada institución participante.

\section{Resultados}

\section{Características sociodemográficas}

En la primera fase del estudio participaron 20 instituciones coordinadas por cardiólogos especialistas en falla cardíaca y se reclutaron 521 pacientes. En la segunda fase del estudio se incluyeron otras 34 instituciones coordinadas por médicos cardiólogos y seis instituciones coordinadas por médicos internistas, y se reclutaron 2007 pacientes (Tabla 1) (Fig. 1).

Se incluyeron en total 2528 pacientes con falla cardiaca agudamente descompensada. El $57.59 \%$ eran hombres y la mediana de edad fue 69 años $(Q 1=59$; Q3 $=78$ ). El $43.83 \%$ estaban casados, el $57.91 \%$ no tenían ningún grado de escolaridad o solo habían cursado primaria incompleta, el $58.43 \%$ pertenecían al régimen social contributivo y el $72.82 \%$ venían del área urbana de sus ciudades. Los departamentos que reclutaron mayor número de pacientes $(>100)$ fueron Cundinamarca (14.32\%), Valle del Cauca (14.28\%), Caquetá $(9.38 \%)$, Atlántico $(9.38 \%)$, Antioquia $(9.22 \%)$, Santander $(7.08 \%)$, Boyacá $(7.00 \%)$ y Risaralda $(4.98 \%)$ (Figs. 2-3), mientras que las ciudades que reclutaron mayor número de pacientes (> 100) fueron Cali (347, $13.73 \%)$, Bogotá $(309,12.22 \%)$, Barranquilla (237, 9.38\%), Florencia (237, 9.38\%), Medellín (207, 8.19\%), Bucaramanga (179, 7.08\%), Pereira (126, 4.98\%) y Tunja (112, 4.43\%). Las capitales de todos los departamentos participantes incluyeron pacientes en el registro, al igual que Rionegro (Antioquia), Duitama (Boyacá), Roldanillo (Valle del Cauca), Chía y Soacha (Cundinamarca) (Fig. 4). Los departamentos con más instituciones participantes fueron Cundinamarca (9), Atlántico (8), Valle del Cauca (8) y Antioquia (5) (Figs. 1-5).

\section{Comorbilidad}

Las enfermedades concomitantes más comunes fueron hipertensión arterial (72.04\%), enfermedad coronaria $(28.08 \%)$, dislipidemia $(25.74 \%)$, diabetes tipo $2(24.66 \%)$, fibrilación atrial (22.28\%), enfermedad pulmonar obstructiva crónica $(17.54 \%)$, insuficiencia renal (17.26\%) y valvulopatía (17.06\%).

\section{Características clínicas}

La etiología más frecuente de la falla cardiaca fue la isquémica $(43.87 \%)$, seguida de la hipertensiva (32.71\%). El $11.83 \%$ de los pacientes tenían una clase funcional NYHA I, el $53.60 \%$ tenían clase II, el $29.87 \%$ clase III y el $4.71 \%$ clase IV. La clasificación ACC/AHA más común fue $\mathrm{D}(90.43 \%)$. El $73.54 \%$ de los pacientes tenían una FEVI reducida (<40\%), el $10.33 \%$ en rango intermedio $(40-50 \%)$ y el $16.13 \%$ preservada (> 50\%). El $22.47 \%$ tenían algún tipo de dispositivo implantable (cardiodesfibrilador implantable [CDI] el 9.93\% y resincronizador más CDI el 5.02\%) (Tabla 2).

\section{Discusión}

El registro RECOLFACA es la primera aproximación que se realiza en nuestro país sobre el perfil clínico y demográfico de los pacientes con falla cardiaca en instituciones de gran parte del territorio nacional. Esta información es de gran relevancia porque permitirá caracterizar la población y servir como base para diseñar políticas públicas que brinden una mejor atención en salud a esta población. Este importante proyecto epidemiológico, realizado por el Capítulo de Falla Cardíaca, Trasplantes e Hipertensión Pulmonar de la Sociedad Colombiana de Cardiología y Cirugía Cardiovascular, es pionero en la evaluación de las enfermedades cardiovasculares en Colombia y, adicionalmente, servirá como modelo para la evaluación de otras enfermedades.

Existen registros internacionales similares que recolectan activamente información, como el registro europeo a largo plazo de insuficiencia cardiaca, el cual ha permitido identificar diferencias en el tratamiento y el pronóstico de la población en diferentes regiones de Europa ${ }^{17}$.

Una de las grandes fortalezas de la información que se obtendrá es el registro de variables sociales, como el tipo de aseguramiento de los pacientes, su procedencia de zonas rurales o urbanas, y el nivel de complejidad de los hospitales donde recibieron atención, 
Tabla 1. Instituciones participantes por departamentos y ciudades (en orden alfabético)

\begin{tabular}{|c|c|c|}
\hline Departamento & Ciudad & Institución \\
\hline Amazonas & Leticia & Rafael Bustamante y Compañía Ltda. \\
\hline \multirow[t]{2}{*}{ Antioquia } & Medellín & $\begin{array}{l}\text { Clínica CardioVid } \\
\text { Clínica El Rosario } \\
\text { Clínica Las Américas } \\
\text { Clínica Medellín }\end{array}$ \\
\hline & Rionegro & Centro Cardiovascular Somer In Care S.A. \\
\hline Atlántico & Barranquilla & $\begin{array}{l}\text { Cardiodiagnóstico S.A. } \\
\text { Cardiología Integral } \\
\text { Centro Científico Asistencial } \\
\text { Centro Cardiológico Procardiocaribe S.A.S. } \\
\text { Clínica de la Costa } \\
\text { Hospital Universidad del Norte } \\
\text { Institución Clínica Iberoamérica } \\
\text { IPS Salud Social }\end{array}$ \\
\hline Bolívar & Cartagena & $\begin{array}{l}\text { Centro Diagnóstico Cardiológico } \\
\text { Unidad Cardiológica de Cartagena }\end{array}$ \\
\hline \multirow[t]{2}{*}{ Boyacá } & Tunja & Clínica Mediláser \\
\hline & Duitama & $\begin{array}{l}\text { Mediagnóstica S.A.S. } \\
\text { Unidad de Diagnostico Cardiovascular Sveins }\end{array}$ \\
\hline Caldas & Manizales & $\begin{array}{l}\text { Clínica Avidanti } \\
\text { Clínica San Marcel-Confa }\end{array}$ \\
\hline Caquetá & Florencia & Clínica Mediláser \\
\hline Cauca & Popayán & $\begin{array}{l}\text { Hospital Universitario San José } \\
\text { Unidad Cardiológica del Cauca Única S.A.S. }\end{array}$ \\
\hline Cesar & Valledupar & Instituto Cardiovascular del Cesar (ICVC) \\
\hline Chocó & Quibdó & Cardiodiagnóstico del Chocó \\
\hline Córdoba & Montería & $\begin{array}{l}\text { Cardio Care Colombia S.A.S. } \\
\text { Hospicardio }\end{array}$ \\
\hline \multirow[t]{3}{*}{ Cundinamarca } & Bogotá & $\begin{array}{l}\text { Clínica Universitaria Colombia Colsanitas } \\
\text { Clínicos IPS } \\
\text { Fundación Clínica Shaio } \\
\text { Fundación Santa Fe } \\
\text { Hospital Militar Central } \\
\text { Hospital Universitario San Ignacio } \\
\text { Hospital Santa Clara }\end{array}$ \\
\hline & Chía & Clínica Chía \\
\hline & Soacha & Hospital Cardiovascular del Niño de Cundinamarca \\
\hline Huila & Neiva & Clínica Mediláser Neiva \\
\hline La Guajira & Riohacha & $\begin{array}{l}\text { Clínica Renacer } \\
\text { Clínica Riohacha }\end{array}$ \\
\hline Magdalena & Santa Marta & Group Salud IPS \\
\hline Meta & Villavicencio & Sociedad Cardiológica Colombiana S.A.S. \\
\hline Nariño & Pasto & $\begin{array}{l}\text { Clínica Nuestra Señora de Fátima } \\
\text { Unimedic IPS }\end{array}$ \\
\hline Norte de Santander & Cúcuta & Hospital Universitario Erasmo Meoz \\
\hline Quindío & Armenia & Centro Cardiovascular \& Diabetes MASSALUD \\
\hline Risaralda & Pereira & $\begin{array}{l}\text { Clínica Los Rosales } \\
\text { Sanidad Policía Nacional Seccional Risaralda }\end{array}$ \\
\hline \multirow[t]{2}{*}{ Santander } & Bucaramanga & Instituto del Corazón de Bucaramanga \\
\hline & Floridablanca & Fundación Cardiovascular de Colombia \\
\hline Sucre & Sincelejo & Hospital Universitario de Sincelejo \\
\hline Tolima & Ibagué & Cardiología Siglo XXI \\
\hline \multirow[t]{2}{*}{ Valle del Cauca } & Cali & $\begin{array}{l}\text { Fundación Valle de Lili } \\
\text { Angiografía de Occidente } \\
\text { Clínica Amiga } \\
\text { Dime Clínica Neuro Cardiovascular } \\
\text { Clínica de Occidente } \\
\text { Clínica Imbanaco } \\
\text { Hospital Universitario del Valle }\end{array}$ \\
\hline & Roldanillo & Hospital San Antonio de Roldanillo \\
\hline
\end{tabular}




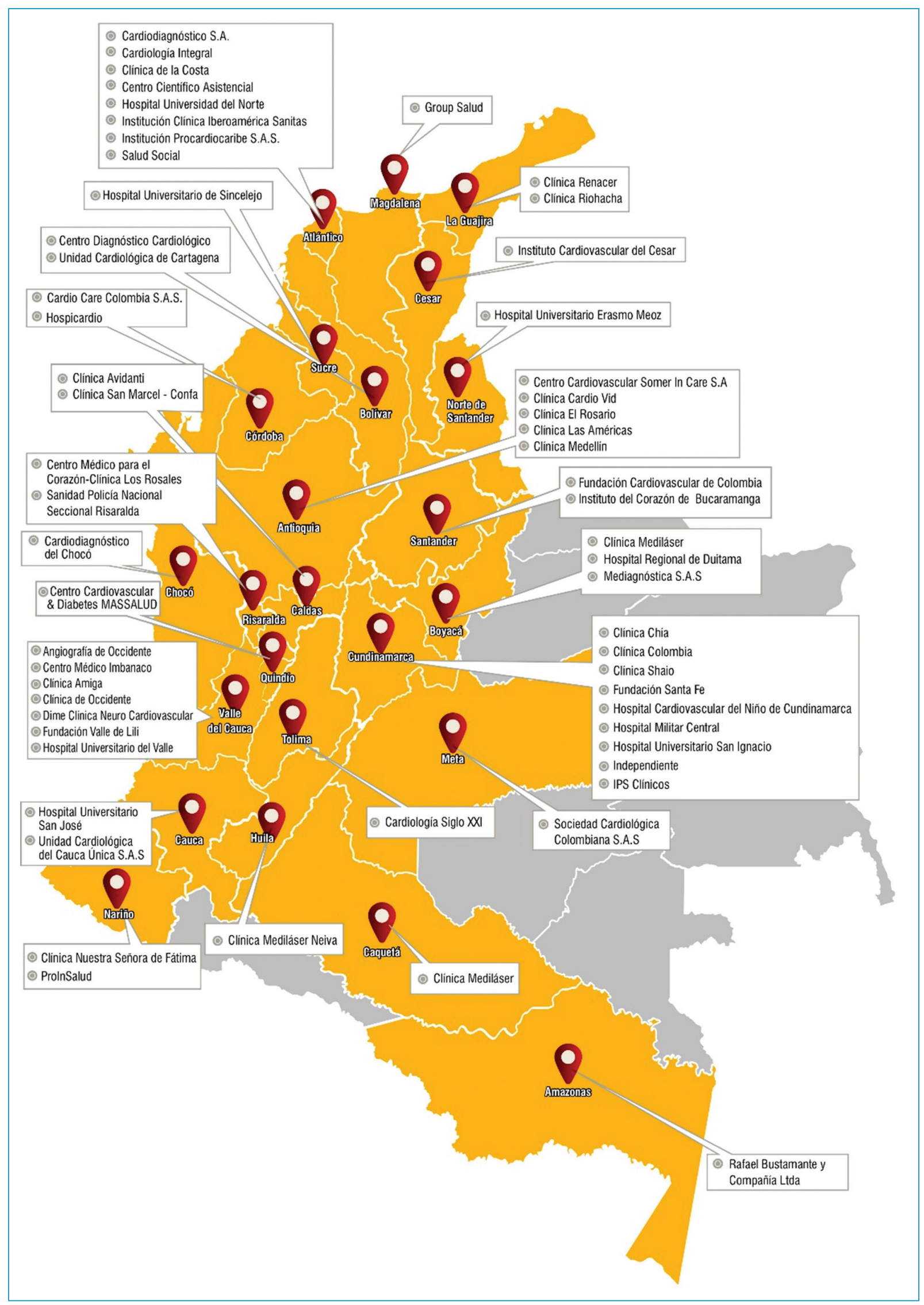

Figura 1. Instituciones participantes en el RECOLFACA por departamentos. 


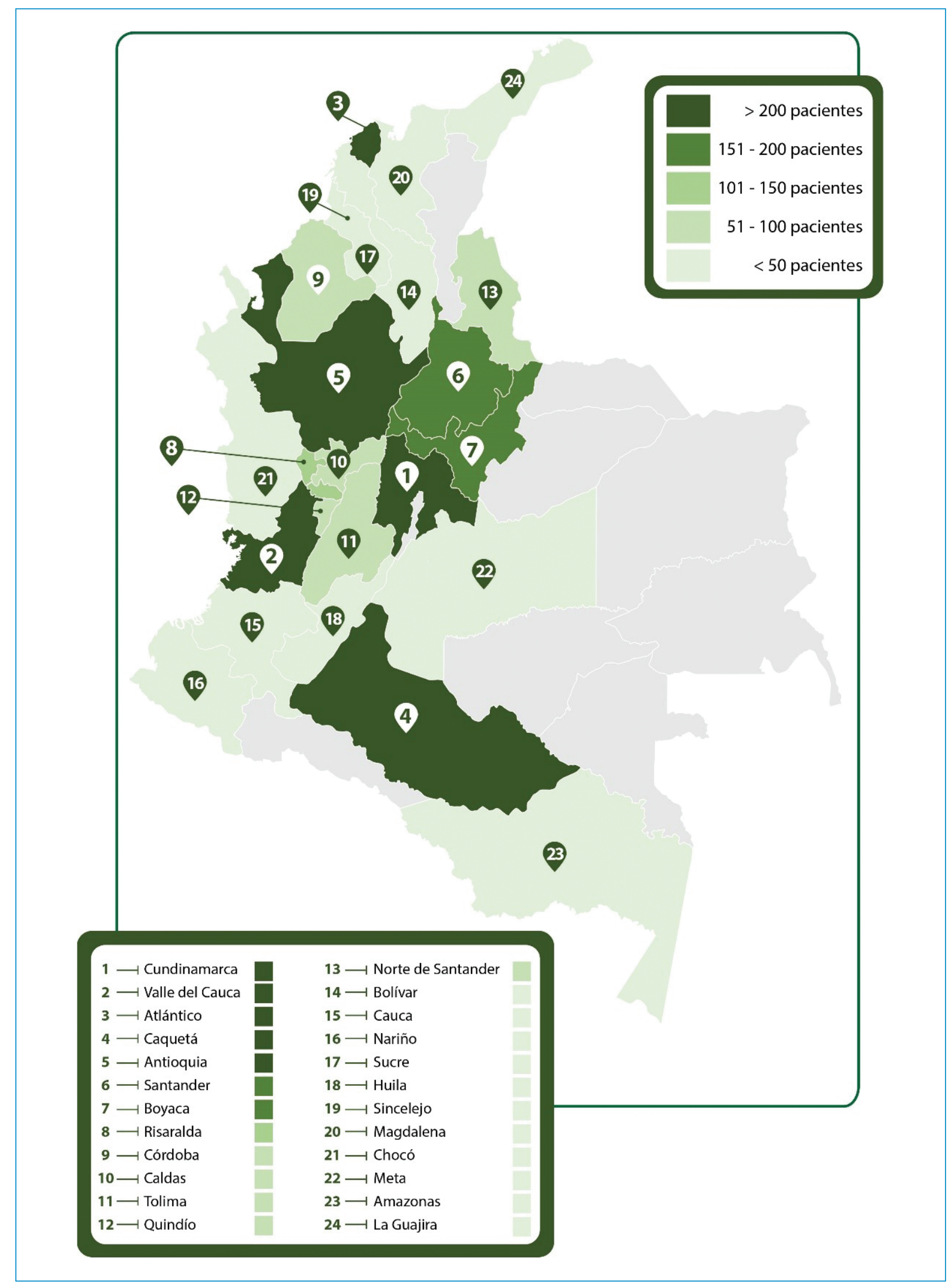

Figura 2. Reclutamiento por departamentos. 
Rev Colomb Cardiol. 2021;28(3)

Tabla 2. Características clínicas $(n=2528)$

\begin{tabular}{|c|c|c|c|}
\hline Variable & n (\%) & Variable & n (\%) \\
\hline Etiología de la falla cardiaca & & PAS $(\mathrm{mmHg})$ & $120(106-134)$ \\
\hline Isquémica & $1112(43,99)$ & Frecuencia cardiaca (I.p.m.) & $72(65-81)$ \\
\hline Hipertensiva & $804(31.80)$ & Radiografía de tórax & \\
\hline Valvular & $331(13.09)$ & Normal & $271(10.72)$ \\
\hline Taquicardiomiopatía & $66(2.61)$ & Anormal & $858(33.94)$ \\
\hline Idiopática & $225(8.90)$ & No se tienen datos & $1399(55.34)$ \\
\hline Metabólica & $16(0.63)$ & Radiografía de tórax & \\
\hline Chagásica & $88(3.48)$ & Cardiomegalia & $730(85.08)$ \\
\hline Tóxica & $13(0.51)$ & Congestión hiliar & $302(35.19)$ \\
\hline Viral & $20(0.79)$ & Derrame pleural & $219(25.52)$ \\
\hline Quimioterapia & $17(0.67)$ & Hallazgos en el ECG & \\
\hline Congénita & $15(0.59)$ & Ritmo sinusal & 869 (64.99) \\
\hline Genética & $13(0.51)$ & Hipertrofia & $192(14.36)$ \\
\hline Periparto & $8(0.32)$ & Trastorno de repolarización & $295(22.06)$ \\
\hline Alcohólica & $2(0.08)$ & Signos de isquemia & $131(9.79)$ \\
\hline Clase funcional NYHA & & Marcapasos & $110(8.22)$ \\
\hline I & $299(11.83)$ & Fibrilación atrial & $237(17.72)$ \\
\hline II & $1355(53.60)$ & Bloqueo auriculoventricular & $75(5.60)$ \\
\hline III & $755(29.87)$ & Onda 0 & $96(7.18)$ \\
\hline IV & $119(4.71)$ & QRS (ms) & \\
\hline Clasificación ACC/AHA & & $<120$ & $833(62.26)$ \\
\hline C & $2390(94.54)$ & $120-130$ & $339(25.34)$ \\
\hline \multirow[t]{2}{*}{ D } & $138(5.46)$ & $130-150$ & $107(8.00)$ \\
\hline & & $>150$ & $59(4.41)$ \\
\hline $\begin{array}{l}\text { Valoración previa FEVI } \\
\text { Sí } \\
\text { No }\end{array}$ & $\begin{array}{c}2200(87.03) \\
328(12.97)\end{array}$ & $\begin{array}{l}\text { Información DDVI } \\
\text { Sí } \\
\text { No }\end{array}$ & $\begin{array}{c}1310(63.84) \\
742(36.16)\end{array}$ \\
\hline $\begin{array}{l}\text { FEVI previa } \\
\text { Reducida }(\leq 40 \%) \\
\text { Medio rango }(41-49 \%) \\
\text { Preservada }(\geq 50 \%)\end{array}$ & $\begin{array}{c}1,661(75.50) \\
207(9.41) \\
332(15.09)\end{array}$ & $\begin{array}{l}\text { DDVI }(\mathrm{mm}) \\
\text { FEVI actual }(\%) \\
\text { Reducida }(\leq 40 \%) \\
\text { Medio rango }(41-49 \%) \\
\text { Preservada }(\geq 50 \%)\end{array}$ & $\begin{array}{l}57(48-65)^{*} \\
1509(73.54) \\
212(10.33) \\
331(16.13)\end{array}$ \\
\hline $\begin{array}{l}\text { Dispositivos previos } \\
\text { Sí } \\
\text { No } \\
\text { Tipo de dispositivo } \\
\text { CDI } \\
\text { Resincronizador + CDI } \\
\text { MPD bicameral } \\
\text { MPD unicameral } \\
\text { Resincronizador }\end{array}$ & $\begin{array}{c}566(22.43) \\
1961(77.57) \\
245(9.69) \\
127(5.02) \\
97(3.82) \\
49(1.94) \\
48(1.90)\end{array}$ & $\begin{array}{l}\text { Hipertensión pulmonar } \\
\text { Sí } \\
\text { No } \\
\text { No se tienen datos } \\
\text { Insuficiencia mitral } \\
\text { Grado II } \\
\text { Grado III } \\
\text { Grado IV } \\
\text { No se tienen datos }\end{array}$ & $\begin{array}{c}892(43.47) \\
947(46.15) \\
213(10.38) \\
\\
883(43.03) \\
342(16.67) \\
178(8.67) \\
649(31.63)\end{array}$ \\
\hline
\end{tabular}

ACC: American College of Cardiology; AHA: American Heart Association; CDI: cardiodesfibrilador implantable; DAV: dispositivo de asistencia ventricular; DDVI: diámetro diastólico de ventrículo izquierdo; ECG: electrocardiograma; FEVI: fracción de eyección del ventrículo izquierdo; NYHA: New York Heart Association; PAS: presión arterial sistólica. 


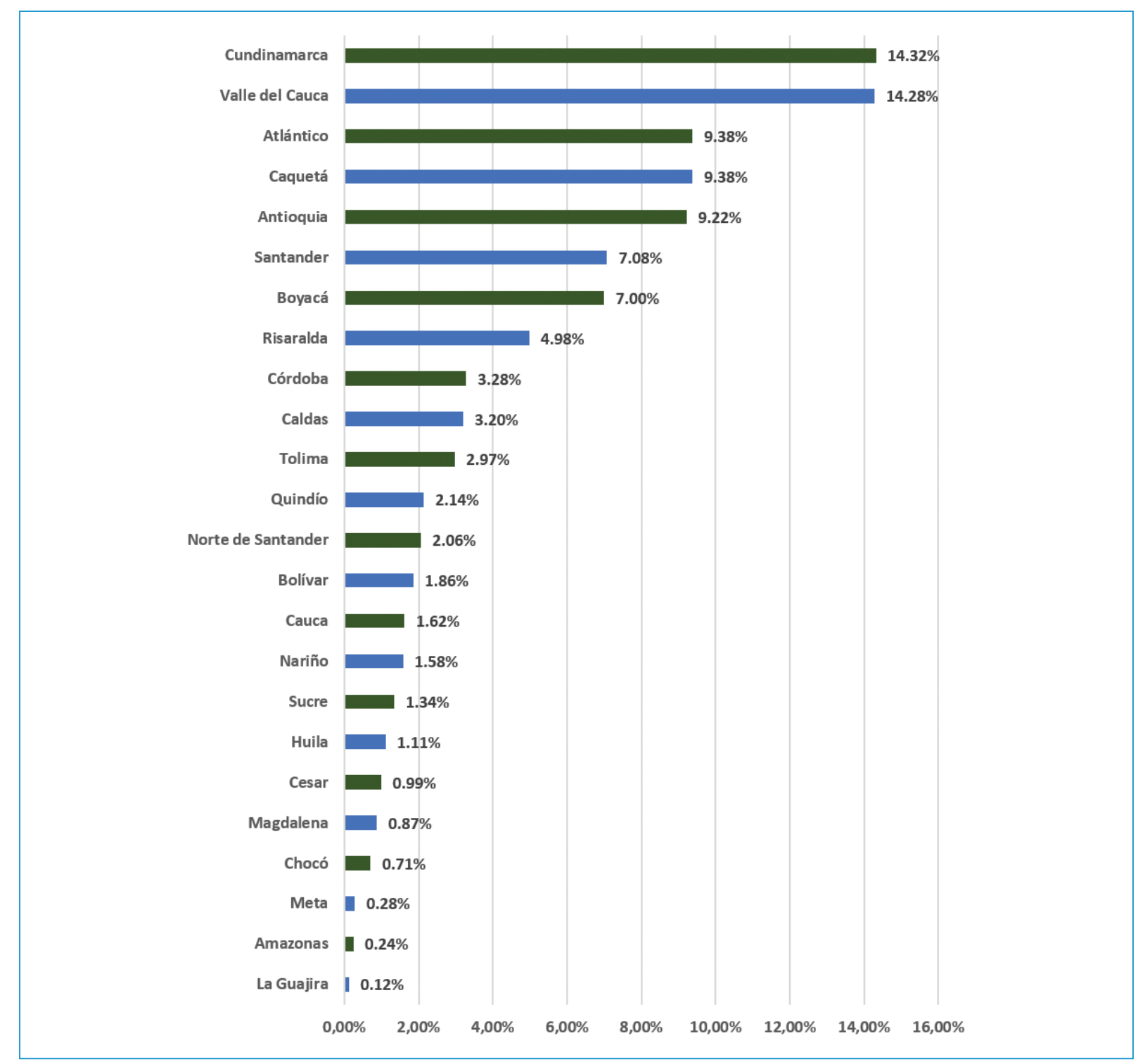

Figura 3. Porcentaje de reclutamiento por departamentos.

pues con ello se identifican barreras en el acceso a los cuidados en salud y sus implicaciones pronósticas. Determinar variables como la rehospitalización y la mortalidad también es indispensable para establecer modelos económicos que permitan medir el impacto de la enfermedad en términos de costos y ser más eficientes en el uso de los recursos del sistema de salud colombiano.

Entre las posibles etiologías de la falla cardiaca, la cardiopatía chagásica es una enfermedad endémica en algunos países, como Colombia, y este grupo de pacientes son parte del objetivo a incluir en el registro. Esta información será prioritaria para caracterizar esta población y también para desarrollar una estrategia integral que incluya medidas de prevención, diagnóstico temprano y tratamiento oportuno que permitan disminuir la morbilidad y la mortalidad asociadas a esta condición.

El Ministerio de Salud y Protección Social de Colombia ha realizado un gran esfuerzo con la elaboración de guías de práctica clínica para el diagnóstico, el tratamiento y la rehabilitación de los pacientes con insuficiencia cardiaca ${ }^{18}$, y la mejor manera de evaluar la adherencia a estas recomendaciones es mediante un registro de la vida real, como RECOLFACA, en el que por primera vez se podrá identificar si se están 


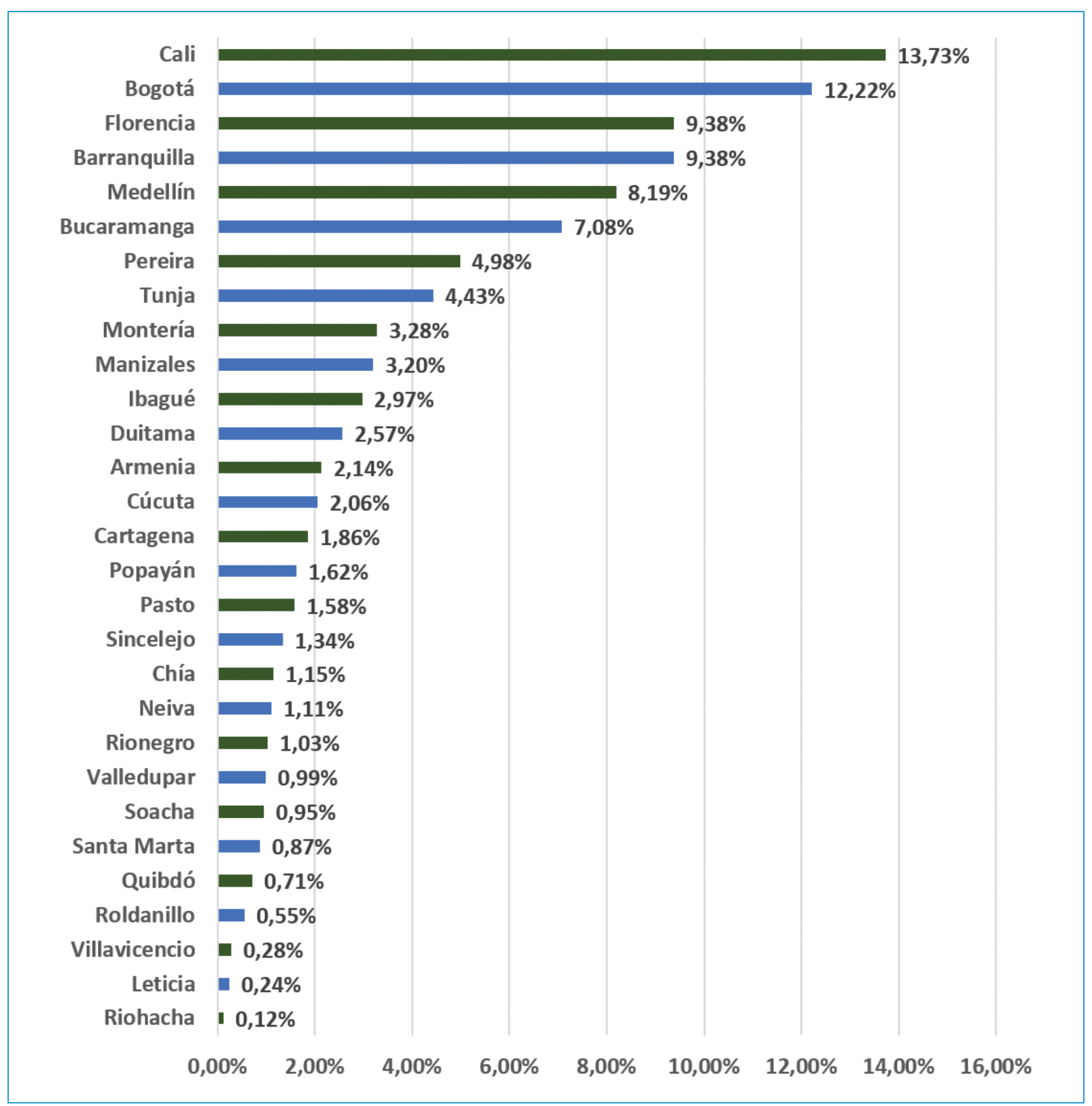

Figura 4. Porcentaje de reclutamiento por ciudades.

prescribiendo los medicamentos y se están realizando las intervenciones que modifican el curso de la enfermedad, y en caso de demostrarse deficiencias en el uso de la terapia médica óptima poder diseñar estrategias de educación médica continua dirigidas a mejorar la práctica clínica y el cuidado de la enfermedad.

La perspectiva de los pacientes sobre el impacto de la enfermedad en su calidad de vida es otro de los aspectos destacables de este registro, ya que pocas enfermedades limitan tanto la esfera física, mental y social de las personas como la falla cardiaca, y por esta razón, la recolección de tales datos permitirá evaluar esta perspectiva que es indispensable para entender el beneficio de los tratamientos que se ofrecen para la enfermedad ${ }^{19}$.

\section{Limitaciones}

La información incluida en el registro puede presentar sesgos de selección por las características de los centros que reclutaron pacientes en el país, en los cuales se incluyeron como investigadores principales especialistas en medicina interna, cardiología y falla 


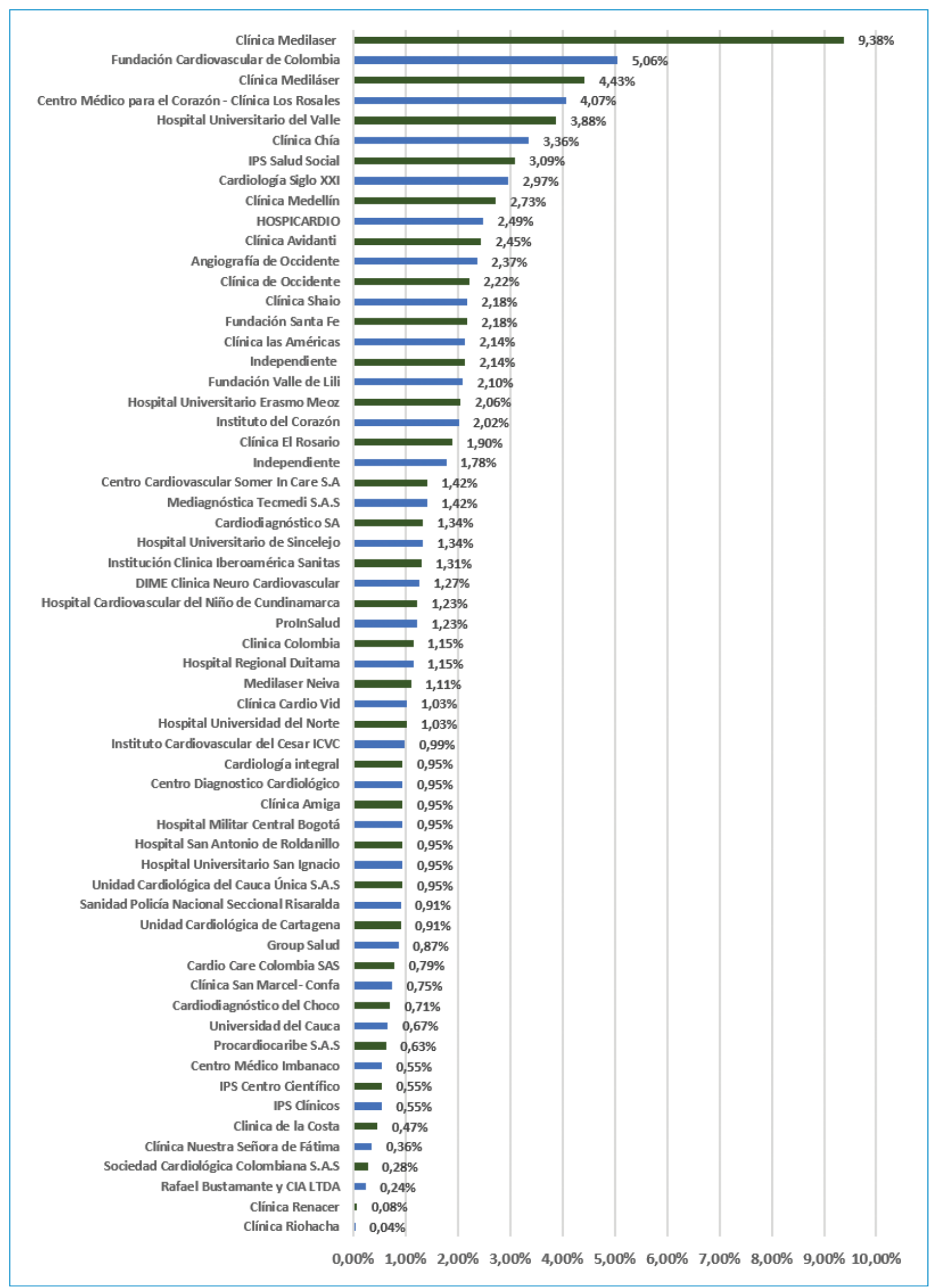

Figura 5. Porcentaje de reclutamiento por instituciones. 
cardiaca, y probablemente no refleje la práctica diaria de otros actores del sistema de salud, como los médicos de atención primaria. La población incluida está conformada por pacientes con historia de descompensación de falla cardiaca y los resultados no reflejan la realidad de los pacientes crónicos sin eventos de descompensación previa, que podrían presentar un curso clínico diferente.

\section{Conflicto de intereses}

Los autores manifiestan no presentar conflictos de intereses.

\section{Responsabilidades éticas}

Protección de personas y animales. Los autores declaran que para esta investigación no se han realizado experimentos en seres humanos ni en animales.

Confidencialidad de los datos. Los autores declaran que han seguido los protocolos de su centro de trabajo sobre la publicación de datos de pacientes.

Derecho a la privacidad y consentimiento informado. Los autores declaran que en este artículo no aparecen datos de pacientes.

\section{Bibliografía}

1. Go AS, Mozaffarian D, Roger VL, Benjamin EJ, Berry JO, Blaha MI, et al. Heart disease and stroke statistics - 2014 update: a report from the American Heart Association. Circulation. 2014;129:e28.

2. Liu L, Eisen HJ. Epidemiology of heart failure and scope of the problem. Cardiol Clin. 2014;32:1-8.

3. Dunlay SM, Redfield MM, Weston SA, Therneau TM, Hall Long K, Shah ND, et al. Hospitalizations after heart failure diagnosis: a community perspective. J Amn Coll Cardiol. 2009;54:1695-702.
4. Heidenreich PA, Albert NM, Allen LA, Bluemke DA, Butler J, Fonarow $\mathrm{GC}$, et al. Forecasting the impact of heart failure in the United States. A policy statement from the American Heart Association. Circ Heart Fail. 2013;6:606-19.

5. Yancy CW, Jessup M, Bozkurt B, Butler J, Casey DE, Drazner MH, et al. 2013 ACCF/AHA guideline for the management of heart failure: a report of the American College of Cardiology Foundation/American Heart Association Task Force on Practice Guidelines. J Am Coll Cardiol. 2013;62:e147-e239.

6. America HFSA. Executive summary: HFSA 2010 comprehensive heart failure practice guideline. J Cardiac Fail. 2010;16:475-539.

7. Gheorghiade M, Pang PS. Acute heart failure syndromes. J Am Coll Cardiol. 2009;53:557-73.

8. Cotter G, Moshkovitz Y, Milovanov O, Salah A, Blatt A, Krakover R, et al Acute heart failure: a novel approach to its pathogenesis and treatment. Eur Heart J. 2002;4:227-34.

9. Tavazzi L, Maggioni AP, Lucci D, Cacciatore G, Ansalone G, Oliva F, et al. Nationwide survey on acute heart failure in cardiology ward services in Italy. Eur Heart J. 2006;27:1207-15.

10. Zannad F, Mebazaa A, Juilliére Y, Cohen-Solal A, Guize L, Alla F, et al. Clinical profile, contemporary management and one-year mortality in patients with severe acute heart failure syndromes: the EFICA study. Eur J Heart Fail. 2006;8:697-705.

11. Cleland L, Swedberg K, Follath F, Komajda M, Cohen-Solal A, Aguilar JC, et al. The EuroHeart Failure survey programme - a survey on the quality of care among patients with heart failure in Europe. Part 1: patient characteristics and diagnosis. Eur Heart J. 2003;24:442-63.

12. Fonarow GC, Abraham WT, Albert NM, Stough WG, Gheorghiade M, Greenberg $\mathrm{BH}$, et al. Factors identified as precipitating hospital admissions for heart failure and clinical outcomes: findings from OPTIMIZE-HF. Arch Int Med. 2008;168:847-54.

13. Hernández-Leiva E. Epidemiología del síndrome coronario agudo y la insuficiencia cardíaca en Latinoamérica. Rev Esp Cardiol. 2011;64:34-43.

14. Observatorio Nacional de Salud del Instituto Nacional de Salud. Boletín 01: La Enfermedad Cardiovascular como principal causa de muerte en Colombia. 2013. [citado noviembre 2020]. Disponible en: https://www.ins. gov.co/Direcciones/ONS/Boletines/boletin_web_ONS/graficas_tablas.html

15. Senior LM, Saldarriaga C, Rendón JA. Descripción clínico-epidemiológica de los pacientes con falla cardíaca aguda que consultan al servicio de urgencias. Acta Med Colomb. 2011;36:125-9.

16. Calderón LE, Satizábal N, Rincón EA, Olaya P, Flórez N, Carrillo DC, et al. Perfiles clínicos y hemodinámicos en pacientes con falla cardíaca aguda. Rev Col Cardiol. 2017;24:448-57.

17. Crespo-Leiro MG, Anker SD, Maggioni AP, Coats AJ, Filippatos G, Ruschitzka F, et al. European Society of Cardiology Heart Failure LongTerm Registry (ESC-HF-LT): 1-year follow-up outcomes and differences across regions. Eur J Heart Fail. 2016;18:613-25.

18. Senior JM, Lugo LH, Díaz JS, Muñoz E, Tamayo N, Saldarriaga C, et al. Guía de práctica clínica para la prevención, diagnóstico, tratamiento y rehabilitación de la falla cardiaca en población mayor de 18 años, clasificación B, C y D. Guía No. 53. Bogotá: Ministerio de Salud y Protección Social; 2016.

19. Coelho R, Ramos S, Prata J, Bettencourt P, Ferreira A, Cerqueira-Gomes M. Heart failure and health related quality of life. Clin Pract Epidemiol Ment Health. 2005;1:19. 


\section{Anexo}

Anexo. Miembros del Grupo Investigador RECOLFACA

Alberto Cadena

Alejandro D. Ochoa-Morón

Alejandro Posada-Bastidas

Alex Rivera-Toquica

Alexis Llamas

Alfonso Muñoz-Velásquez

Álvaro H. Rodríguez-Cerón

Andrés F. Buitrago

Ángel A. García-Peña

Argemiro R. Martínez-Carvajal

Armando Alcalá-Hernández

Balkis Rolong

Carlos A. Arias-Barrera

Carlos A. Plata-Mosquera

Carlos A. Rentería-Asprilla

Clara I. Saldarriaga-Giraldo

Claudia V. Anchique

Diego H. Hoyos-Ballesteros

Edgar E. Castro-0sorio

Eduardo J. Echeverri-Navarrete

Elkin G. Ramírez-Puentes

\section{Erika Martínez}

Fernán Mendoza-Beltrán

Fernando Manzur-Jatin

Fernando Rivera-Toquica

Gary A. Baquero-Lozano

Guillermo Trout-Guardiola

Gustavo A. Moreno-Silgado

Hugo E. Osorio-Carmona

Jannes Buelvas-Herazo

José I. Mosquera-Jiménez

José L. Accini-Mendoza

Juan A. Cerón

Juan A. Sandoval-Luna
Clínica de la Costa, Barranquilla, Colombia

Instituto Cardiovascular del Cesar ICVC, Cesar, Colombia

Centro Cardiovascular Somer In Care, Rionegro (Antioquia), Colombia

Centro Médico para el Corazón, Clínica Los Rosales, Universidad Tecnológica de Pereira, Pereira, Colombia

Clínica Las Américas, Medellín, Colombia

Institución Clínica Iberoamérica Sanitas, Barranquilla, Colombia

Procardio-Hospital Cardiovascular del Niño de Cundinamarca, Cundinamarca, Colombia

Fundación Santa Fe, Bogotá, Colombia

Hospital Universitario San Ignacio, Pontificia Universidad Javeriana, Bogotá, Colombia Hospital San Antonio de Roldanillo, Valle del Cauca, Colombia

Centro Cardiología Procardiocaribe S.A.S., Barranquilla, Colombia

Cardiología Integral, Barranquilla, Colombia

Clínica Universitaria Colombia Colsanitas, Bogotá, Colombia

Dime Clínica Neuro Cardiovascular, Cali, Colombia

Cardiodiagnóstico del Chocó, Choco, Colombia

Clínica Cardio Vid, Medellín, Colombia

Mediagnóstica, Duitama (Boyacá), Colombia

Centro Cardiovascular \& Diabetes MASSALUD, Armenia, Colombia

Clínica San Marcel-Confa, Manizales, Colombia

Clínica de Occidente, Cali, Colombia

Sociedad Cardiológica Colombiana S.A.S., Villavicencio (Meta), Colombia

Clínica Iberoamérica, Salud Social, Barranquilla, Colombia

Fundación Clínica Shaio, Bogotá, Colombia

Centro Diagnostico Cardiológico, Cartagena, Colombia

Sanidad Policía Nacional Seccional Risaralda, Pereira, Colombia

Hospital Santa Clara, Bogotá, Colombia

Groupsalud IPS, Santa Marta, Colombia

Hospicardio, Montería, Colombia

Clínica Medilaser Neiva, Neiva, Colombia

Cardiodiagnóstico, Barranquilla, Colombia

Unidad Cardiológica del Cauca Única S.A.S., Cauca, Colombia

IPS Centro Científico Asistencial S.A.S., Barranquilla, Colombia

Unimedic IPS, Hospital Universitario Departamental de Nariño, Pasto, Colombia

Cardiología Siglo XXI, Ibagué, Tolima, Colombia 
Rev Colomb Cardiol. 2021;28(3)

Anexo. Miembros del Grupo Investigador RECOLFACA (Continuación)

\begin{tabular}{|c|c|}
\hline Alberto Cadena & Clínica de la Costa, Barranquilla, Colombia \\
\hline Juan C. García & Hospital Universidad del Norte, Soledad (Atlántico), Colombia \\
\hline Juan C. Ortega-Madariaga & Hospital Universitario Erasmo Meoz, Cúcuta (Norte de Santander), Colombia \\
\hline Juan D. López-Ponce de León & Fundación Valle de Lili, Cali, Colombia \\
\hline Juan F. Carvajal-Estupiñan & Instituto del Corazón de Bucaramanga, Bucaramanga, Colombia \\
\hline Julián R. Lugo-Peña & Clínicos IPS, Bogotá, Colombia \\
\hline Julián Vanegas-Eljach & Instituto Cardiovascular Colombiano, Manizales, Colombia \\
\hline Lisbeth N. Morales-Rodríguez & Clínica Medilaser, Tunja, Colombia \\
\hline Luis E. Echeverria & Fundación Cardiovascular de Colombia, FCV, Floridablanca-Santander, Colombia \\
\hline Luis M. Ávila-Barros & Clínica Riohacha, Riohacha (Guajira), Colombia \\
\hline Luis Silva-Díaz-Granados & Clínica Chía, Cundinamarca (Chía), Colombia \\
\hline Luz C. Zarate-Correa & Centro Médico Imbanaco, Hospital Universitario del Valle, Cali, Colombia \\
\hline Marco A. De León-Espitia & Cardio Care Colombia S.A.S. - Clínica Zayma S.A.S., Montería, Colombia \\
\hline Mario H. Zarama-Márquez & Clínica Nuestra Señora de Fátima, San Juan de Pasto (Nariño), Colombia \\
\hline Nelly Velásquez-López & Clínica Medellín de Occidente, Medellín, Colombia \\
\hline Nelson A. López Garzón & Hospital Universitario San José, Popayán, Colombia \\
\hline Nelson E. Murillo-Benítez & Angiografía de Occidente, Cali, Colombia \\
\hline Óscar A. Pacheco-Jiménez & Hospital Militar Central, Bogotá, Colombia \\
\hline Óscar S. Rincón-Peña & Unidad de Diagnóstico Cardiovascular Sveins, Duitama (Boyacá), Colombia \\
\hline Patricia Rodríguez-Gómez & Unidad Cardiológica de Cartagena, Cartagena, Colombia \\
\hline Rafael I. Bustamante-Urzola & Rafael Bustamante y Compañía Ltda., Leticia (Amazonas), Colombia \\
\hline Ricardo Gómez-Palau & Clínica Amiga, Cali, Colombia \\
\hline Rolando Palacio & Clínica Renacer, Riohacha (Guajira), Colombia \\
\hline Sebastián Campbell-Quintero & Clínica Medilaser, Florencia (Caquetá), Colombia \\
\hline Silfriedo Arrieta-González & Hospital Universitario de Sincelejo, Sincelejo (Sucre), Colombia \\
\hline Viviana Quintero-Yepes & Clínica El Rosario, Medellín, Colombia \\
\hline
\end{tabular}

